\title{
Cambios tisulares y celulares por tabaquismo en mucosa bucal clínicamente sana: Estudio clínico transversal
}

\section{Tissue and cellular changes related to smoking in clinically healthy oral mucosa: A cross sectional study}

\author{
Golaszewski AM*, Díaz N*, Villarroel-Dorrego M*
}

\section{RESUMEN}

El presente estudio tuvo como propósito determinar los cambios tisulares y celulares que ocasiona el tabaquismo en la mucosa bucal de aspecto normal.

Metodología: Se estudiaron 30 pacientes con edades comprendidas entre los 19 y los 29 años de edad, divididos en 3 grupos de acuerdo a su estatus tabáquico. De cada individuo se tomó una muestra de mucosa bucal aparentemente sana ubicada distal a la zona donde se realizó la incisión para la exodoncia de un tercer molar, la cual fue procesada y teñida con HEE. Las mucosas fueron evaluadas bajo microscopio de luz y los datos analizados estadísticamente.

Resultados: El grupo de los individuos no fumadores presentó en un $100 \%$ epitelio paraqueratinizado a diferencia del grupo de individuos con tabaquismo que mostró en un $90 \%$ ortoqueratinización $(p=0,0001)$. El estrato basal se presentó intacto en el 100\% del grupo de individuos no fumadores mientras que en el $60 \%$ de los fumadores se observó duplicado $(p=0,003)$. El infiltrado inflamatorio fue mayor en los fumadores pero no estadísticamente significativo, a diferencia de la vascularización que se observó estadísticamente disminuida en este grupo.

Conclusión: La mucosa bucal en fumadores muestra numerosos cambios tisulares y celulares que pueden conllevar al desarrollo de lesiones malignas. La ausencia de cambios clínicos no implica que no exista el riesgo de transformación carcinogénica.

Palabras clave: Tabaquismo, mucosa bucal, displasia epitelial, respuesta inmunológica.

\section{SUMMARY}

The constant contact of cigarette smoke and its components with the oral mucosa leads to a series of microscopic architectural changes of the mucosa.The aim of the study was to determine tissue and cellular changes caused by smoking in the oral mucosa of normal appearance.

Methods: 30 patients aged between 19 and 29 years of age were included in the study. Patients were divided into 3 groups according to their smoking status. A sample of oral mucosa was taken during extraction of third molar, and then processed and stained for HEE. Biopsies were evaluated under light microscope and the data analyzed statistically.

Results: $100 \%$ of non-smokers showed parakeratinized epithelia and orthokeratin was observed in $90 \%$ of smoker cases $(p=0.0001)$. Basal layer was preserved in all healthy individuals, contrary to $60 \%$ of smokers showed basal hyperplasia $(p=0.003)$. Inflammatory infiltrated was observed augmented in smokers but difference was not statically significant. However, in this group number of blood vessels was statistically decreased.

* $\quad$ Facultad de Odontología, Instituto de Biomedicina. Universidad Central de Venezuela. 
Conclusions: Oral mucosa of tobacco users shows tissue and cellular changes which may lead to malignant development. Carcinogenic risk may be present although absence of clinical changes.

Key words: Smoking, oral mucosa, epithelial dysplasia, immunological response.

Fecha de recepción: 3 de febrero de 2013.

Aceptado para publicación: 22 de febrero de 2013.

Golaszewski AM, Díaz N, Villarroel-Dorrego M. Cambios tisulares y celulares por tabaquismo en mucosa bucal clínicamente sana: Estudio clínico transversal. Av. Odontoestomatol 2015; 31 (6): 363-370.

\section{INTRODUCCIÓN}

El tabaquismo es una enfermedad adictiva de tipo crónica con numerosas repercusiones en la salud general y bucal del individuo ocasionada por la absorción de la nicotina y sus subproductos a través de la inhalación del tabaco contenido en el cigarrillo (7).

La mucosa bucal se comporta como una membrana semipermeable que entra en contacto con numerosas sustancias, entre ellas los componentes presentes en el cigarrillo (1-5). La nicotina es absorbida por la mucosa bucal, rasgo importante a tomar en cuenta en el tabaquismo, este contacto directo y de mediano plazo con las sustancias contenidas en el cigarrillo y sus subproductos metabólicos conlleva a una serie de cambios estructurales en la arquitectura histológica de la mucosa bucal $(1,2,4,6)$.

Existen evidencias que existen similitudes entre el efecto del tabaquismo en el epitelio bronquial y la expresión genética en respuesta al tabaquismo en el epitelio de la mucosa bucal, como por ejemplo la alta expresión de los genes que codifican la enzima P450 tanto a nivel bronquial como a nivel bucal de pacientes con tabaquismo; lo cual conlleva a deducir que los cambios histopatológicos pueden ser similares en ambos tejidos $(13,14)$.

Algunos autores mencionan que los cambios observados en la mucosa bucal sana como producto del tabaquismo son hiperqueratosis, hiperparaqueratosis, hiperplasia epitelial, vascularización aumentada a nivel subepitelial dentro del tejido conectivo, promoción de la respuesta inflamatoria evidenciada en un incremento de la cantidad de células por campo
(6, 9-12). Este desequilibrio trae como consecuencia la aparición de desórdenes potencialmente malignos visibles clínicamente, sin embargo previo a la aparición de estas lesiones la mucosa bucal se observa intacta mas no a nivel microscópico.

El objetivo del presente trabajo fue determinar los cambios microscópicos producidos por el tabaquismo en la mucosa bucal aparentemente sana.

\section{MATERIALES Y MÉTODOS}

\section{Población}

Fueron incluidos 30 individuos con edades comprendidas entre 19 y 29 años que acudían al Servicio de Odontología para la exodoncia de los terceros molares. Esta población fue subdividida en tres grupos tomando en cuenta el estatus tabáquico, quedando agrupados de la siguiente manera: 10 individuos que padecían tabaquismo activo (pacientes con consumo activo de cigarrillos de 6 meses o más), 10 individuos que padecían tabaquismo pasivo (individuos en contacto directo diario con un familiar o amigo/ a con tabaquismo activo por un tiempo no menor a 6 meses continuos) y finalmente un grupo de 10 individuos sanos sin contacto conocido o directo con el humo del cigarrillo/tabaco).

\section{Recolección de datos}

A cada paciente le fue realizada una historia clínica exhaustiva que sirvió para determinar la ausencia de cualquier lesión clínica presente en cavidad bucal, establecer el grado de adicción o la relación del pa- 
ciente con el tabaquismo, establecer la presencia del tercer molar totalmente incluido.

Después de la firma de consentimiento informado, se tomó un fragmento de mucosa bucal aparentemente sana no menor a $1 \mathrm{~cm}$ de tamaño aproximadamente de la zona retromolar de cada paciente sometido a la exodoncia de terceros molares completamente retenidos.

Los fragmentos de mucosa bucal fueron lavados con solución fisiológica y posteriormente se colocaron en formol buffer $10 \%$. Se realizaron los pasos habituales de preparación histológica de tejido blando para la tinción de hematoxilina/eosina (fijación, deshidratación, aclaramiento, inclusión, corte, tinción y montaje).

\section{Variables cuantificadas}

- Tipo de queratinización del epitelio.

- Características del estrato basal del epitelio.

- Cantidad, tipo y ubicación del infiltrado inflamatorio en el corion.

- Cantidad de vasos sanguíneos en el corion.

\section{Análisis estadístico}

La semicuantificación del número de células inflamatorias y vasos sanguíneos fue calculada en 10 campos, los cuales nunca se repitieron. Los valores fueron promediados y calculada la media \pm desviación estándar.

Las variables, según su naturaleza, fueron comparadas usando chi $^{2}$ de Pearson, confirmado por el test exacto de Fisher, y análisis de varianza. El nivel de significancia fue establecido en 0,05. Valores p menores a 0,05 fueron considerados estadísticamente significativos.

\section{RESULTADOS}

\section{Tipo de queratinización}

Tal como lo muestra la figura 1, el grupo de los individuos sanos presentó, en un 100\%, epitelio pa-

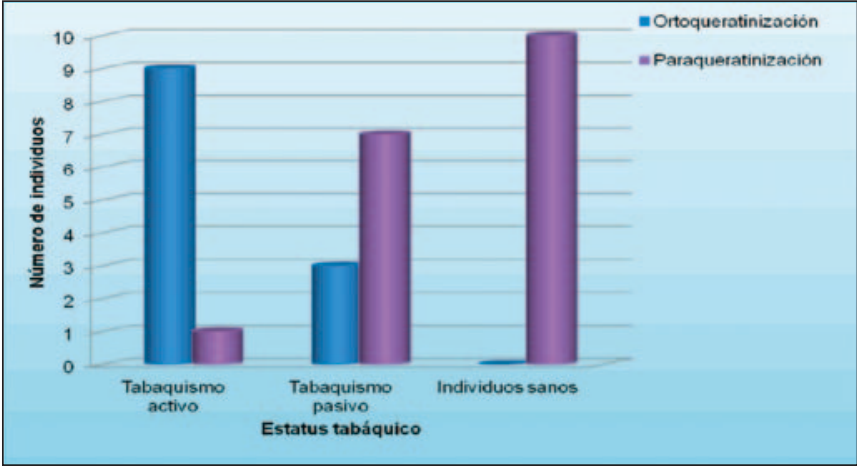

Fig. 1. Distribución de la población de acuerdo al estatus tabáquico y el tipo de queratinización del epitelio de la mucosa bucal.

raqueratinizado (Fig. 2), a diferencia del grupo de individuos con estatus de tabaquismo activo que mostró en un $90 \%$ ortoqueratinización del epitelio (Fig. 3). El grupo de individuos con tabaquismo pasivo mostró un comportamiento similar al grupo de individuos sanos ya que en $70 \%$ de los individuos se observó el epitelio de tipo paraqueratinizado (Fig. 1). El grado de queratinización del epitelio mostró ser una variable con un alto grado de correlación con el estatus tabáquico $(p=0,0001585)$.

\section{Características del estrato basal}

El estrato basal se presentó intacto en el $100 \%$ del grupo de individuos sanos mientras que, en el gru-

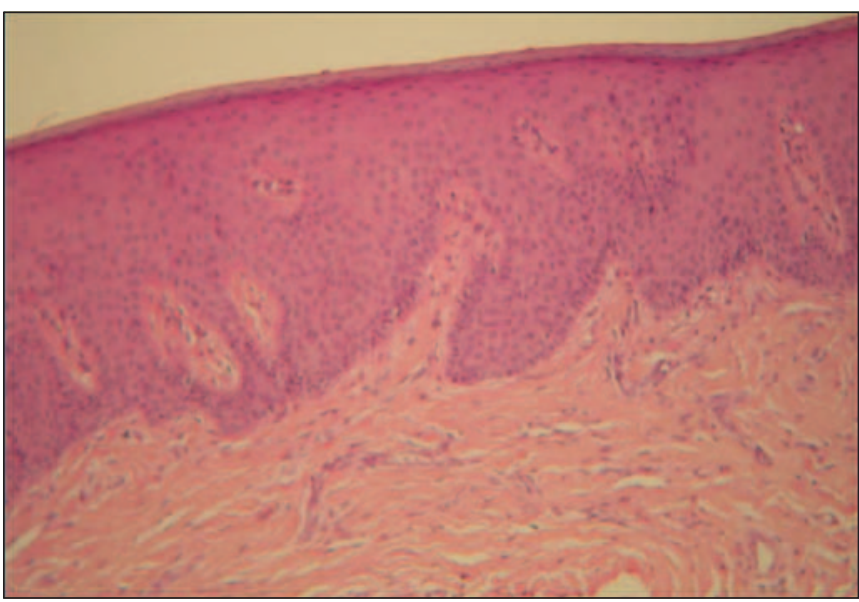

Fig. 2. Microfotografía de individuo sano en donde se observa muestra de un epitelio plano estratificado paraqueratinizado. 


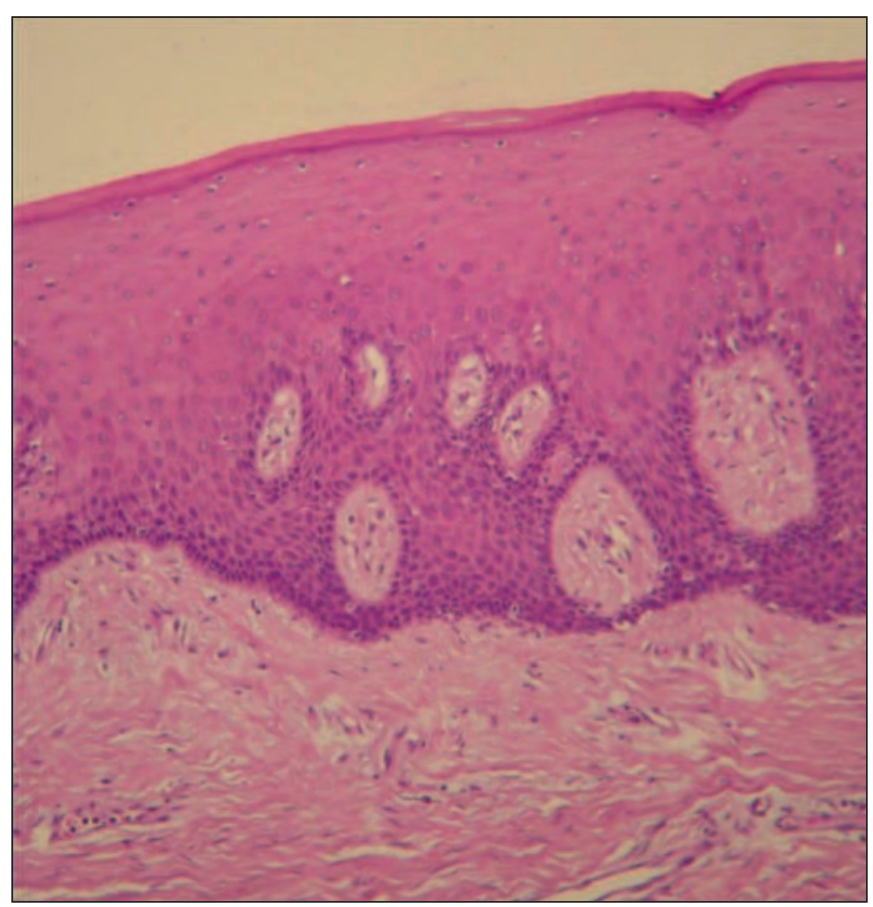

Fig. 3. Microfotografía de paciente con estatus de tabaquismo activo en donde se muestra un epitelio plano estratificado ortoqueratinizado.

po de individuos con estatus de tabaquismo activo, se denotó que el $60 \%$ (6 pacientes) mostraban un estrato basal duplicado (Fig. 4). En relación al grupo de individuos con estatus de tabaquismo pasivo tuvo un comportamiento similar a los individuos no fumadores, ya que sólo el $10 \%$ (1 paciente) presentó un epitelio con el estrato basal duplicado. La duplicación del estrato basal fue significativamente

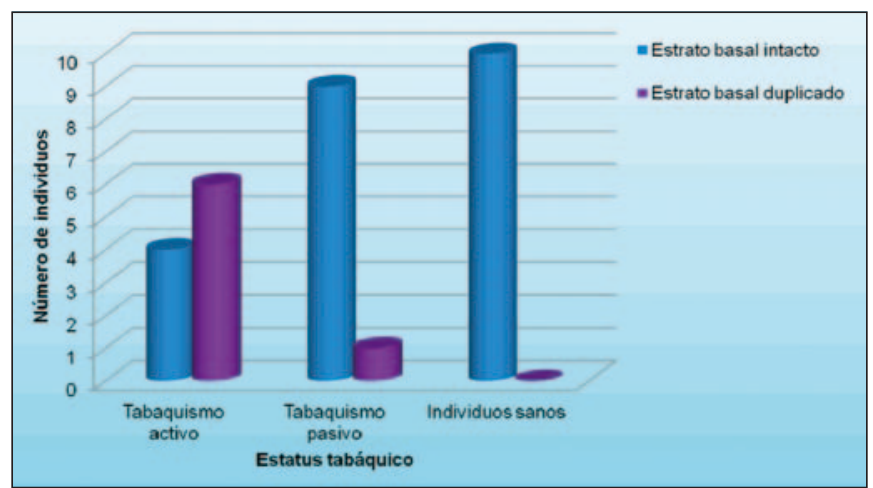

Fig. 4. Distribución de la población de acuerdo al estatus tabáquico y las características del estrato basal del epitelio de la mucosa bucal. mayor en los pacientes con tabaquismo activo $(\mathrm{p}=$ 0,003).

\section{Cantidad, tipo y ubicación del infiltrado inflamatorio}

Infiltrado inflamatorio crónico fue observado en todos los casos, independientemente de su estatus tabáquico, conformado principalmente por linfocitos y plasmocitos. El grupo de individuos con estatus de tabaquismo activo mostró una media de 49,36 \$31,11 células/campo de infiltrado inflamatorio linfoplasmocitario. En el grupo de individuos con tabaquismo se observó una media similar a los fumadores activos (47,70 $\pm 17,78$ células/campo), y aunque la media de células inflamatorias en los individuos sanos fue menor (36,94 13,92 células/campo) no se observaron diferencias estadísticamente significativas ( $p=0,4097)$ (Fig. 5).

En el $90 \%$ (9 pacientes) de los fumadores activos, el infiltrado inflamatorio fue localizado en la profundidad del corion o en toda la extensión del mismo, a diferencia del grupo de individuos sanos $(p=0,000467)$ en donde el infiltrado inflamatorio de todas las muestras fue supeditado a la zona subepitelial, perivascular o ambas (Fig. 6). El grupo de individuos con estatus de tabaquismo pasivo se comportó de forma similar a los fumadores activos ya que en $80 \%$ de los casos el infiltrado inflamatorio se ubicó en toda la extensión del corion.

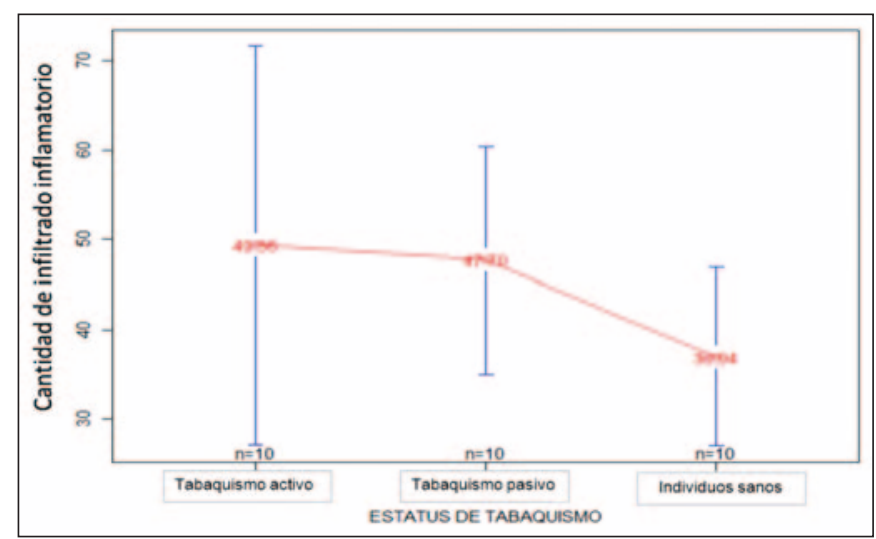

Fig. 5. Distribución de la población de acuerdo al promedio e intervalo de confianza de la cantidad de infiltrado inflamatorio presente en el corion de la mucosa bucal por estatus tabáquico. 


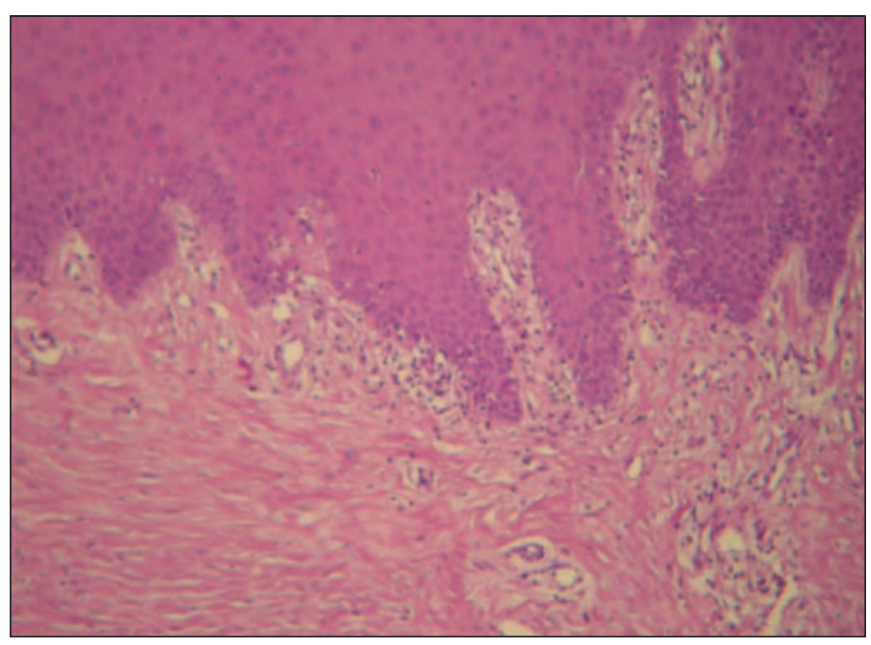

Fig. 6. Microfotografía de individuo sano donde se observa infiltrado inflamatorio localizado a nivel subepitelial y perivascular.

\section{Cantidad de vasos sanguíneos en el corion}

En las figuras 7 a 9, se puede observar cómo el grupo de individuos con estatus de tabaquismo activo mostró un valor medio de vasos sanguíneos $(5,89 \pm 0,96$ vasos/campo) significativamente menor $(p=0,02536)$ que el grupo de individuos con estatus de tabaquismo pasivo $(6,79 \pm 1,75$ vasos/campo) y el grupo de individuos no fumadores (7,93 $\pm 1,83$ vasos/campo).

\section{DISCUSIÓN}

El tabaquismo bien sea activo o pasivo ocasiona daños en la salud bucal, los cuales pueden iniciarse

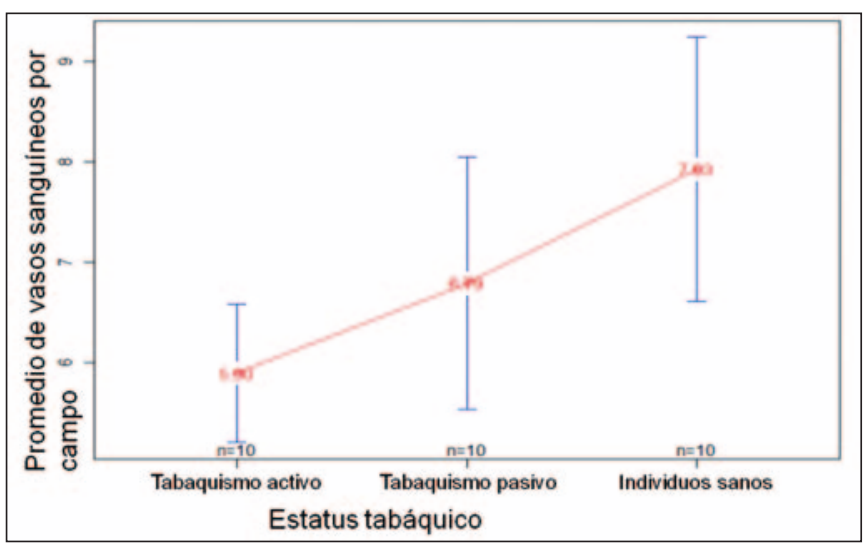

Fig. 7. Distribución de la población de acuerdo al promedio e intervalo de confianza de la cantidad de vasos sanguíneos presentes en el corion de la mucosa bucal por estatus tabáquico.

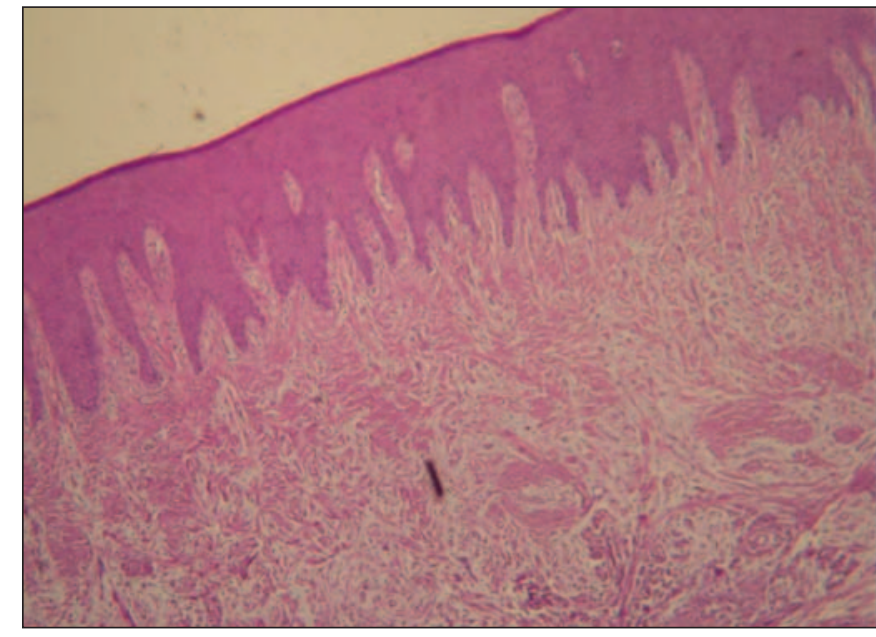

Fig. 8. Microfotografía que muestra la cantidad de vasos sanguíneos presentes en el corion de la mucosa bucal de un individuo con tabaquismo activo.

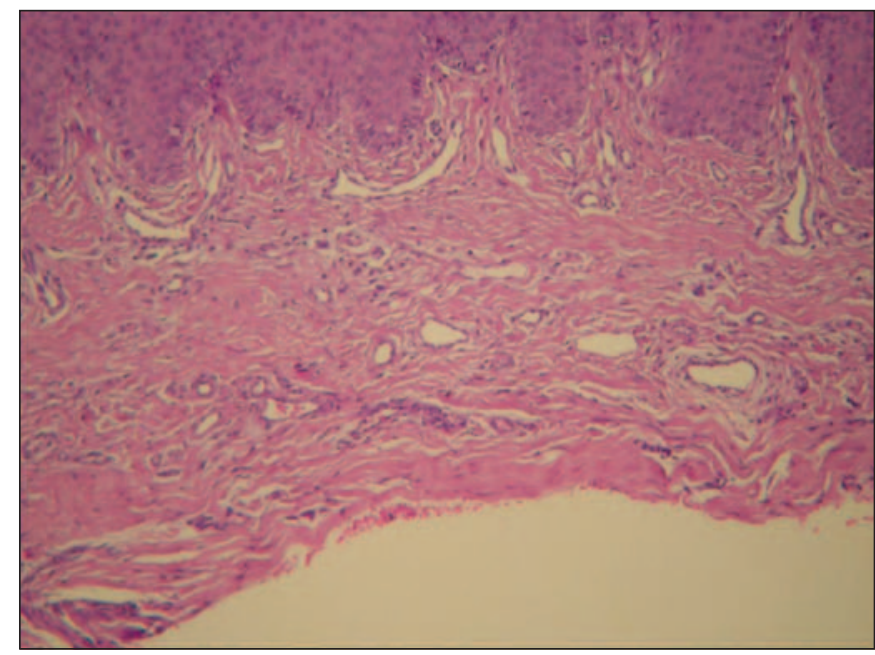

Fig. 9. Microfotografía que muestra la cantidad de vasos sanguíneos presentes en el corion de la mucosa bucal de un individuo sano.

como cambios microscópicos precoces previos a la aparición de lesiones clínicamente visibles. Entre esos cambios se puede mencionar en primer lugar la transformación del tipo de queratinización del epitelio de la mucosa bucal. Este estudio coincide con aquellos que aseveran que se produce un cambio en el tipo de queratinización del epitelio de la mucosa bucal en pacientes con tabaquismo producido posiblemente por alteraciones en el ciclo celular de los queratinocitos lo que conlleva a un grado de queratinización diferente $(10,11)$. Otros trabajos han mostrado más 
que un cambio del tipo de queratinización un aumento del espesor de la capa de paraqueratina en la mucosa de fumadores (12).

La queratina es una barrera impermeable a muchas sustancias. El cambio en el tipo de epitelio de paraqueratinizado a ortoqueratinizado presente en la mucosa de revestimiento de fumadores pareciera ser un mecanismo protector que se genera en respuesta a los productos provenientes del humo del cigarrillo y a los múltiples metabolitos que son secretados a nivel salival que están en contacto directo con la mucosa, de esta manera se intenta frenar el ingreso de estas sustancias a través del epitelio.

Otro de los aspectos tisulares afectados por el tabaquismo es la arquitectura del estrato basal. Este estudio sostiene que existe una fuerte relación entre los cambios presentes en el estrato basal del epitelio y el estatus tabáquico. Los fumadores poseen un riesgo mayor a presentar cambios patológicos tales como displasia epitelial, iniciándose posiblemente con la duplicación de la capa basal (12). Este cambio en la arquitectura epitelial representa otra respuesta en la cinética celular de la mucosa bucal que asegura una barrera de mayor grosor ante los productos del tabaco y sus derivados.

El tabaquismo, por otra parte, promueve la respuesta inflamatoria local de la mucosa bucal (19-21). Interesantemente, se observó un patrón diferencial de ubicación del infiltrado inflamatorio dependiendo del estatus tabáquico, lo que también es indicativo de un fenómeno adaptativo ante una agresión.

El aumento en el número de células inflamatorias y el cambio de su disposición en el tejido bucal de pacientes con tabaquismo podría deberse a una activación local contra alteraciones producidas en el epitelio. Se han observado cambios en la ultraestructura de los linfocitos e inclusive anergia de las células $T$ de fumadores (22-24) lo cual pudiera de alguna forma explicar el desarrollo de lesiones malignas en la mucosa bucal asociada a tabaquismo a pesar de la presencia de un abundante infiltrado inflamatorio local.

En relación a la angiogénesis, se observa un descenso importante de la cantidad de vasos sanguíneos en la mucosa bucal de individuos con tabaquismo activo (24). Sin embargo, existen publicaciones que han probado un aumento en la vascularización a nivel subepitelial dentro del corion en fumadores (13), contrario a los resultados de este estudio. Esta angiogénesis aumentada en fumadores, ha sido descrita como "anormal" o "patológica" contribuyendo a la etiología del cáncer y arteriosclerosis, entre otras enfermedades (25).

Está claro que los productos del tabaco producen un efecto vasoconstrictor en los tejidos, la nicotina es capaz de inhibir la producción de citoquinas antiinflamatorias y quimiotácticas en el endotelio (26), deteriorando el mecanismo inmunológico de defensa contra los cambios epiteliales de la mucosa bucal y finalmente permitiendo a las células neoplásicas epiteliales burlar la vigilancia inmunológica e invadir los tejidos subyacentes.

\section{BIBLIOGRAFÍA}

1. Yildiz D. Nicotine, its metabolism and an overview of its biological effects. Toxicon 2004;43: 619-32.

2. Willems E, Rambali B, Vleeming W, Opperhuizen A, van Amsterdam J. Significance of ammonium compounds on nicotine exposure to cigarette smokers. Food Chem. Toxicol 2006;44(5):67888.

3. Squier CA, Mantz MJ, Wertz PW. Effect of menthol on the penetration of tobacco carcinogens and nicotine across porcine oral mucosa ex vivo. Nicotine Tob Res 2010;12(7): 763-7.

4. Warnakulasuriya S, Sutherland G, Scully C. Tobacco, oral cancer, and treatment of dependence. Oral Onco. 2005;41(3):244-60.

5. Adrian C, Olin H, DalHoff K, Jacobsen J. In vivo human buccal permeability of nicotine. Int $J$ Pharm 2006;311(1-2):196-202.

6. Caldeira E, Fabrega C, Padovani C, Camilli J, García P, Alves V. Morphological alterations in the epithelium of the oral mucosa of rats (Rattus 
norvegicus) submited to long-term systemic nicotine treatment. Arch Oral Biol 2007;52(1): 83-9.

7. Mûller F, Wehbe L. Smoking and smoking cessation in Latin America: a review of the current situation and available treatments. Int J Chron Obstruct Pulmon Dis 2008;3(2):285-93.

8. Shearman E, Fallon S, Sershen H, Lajtha A. Nicotine-induced monoamine neurotransmitter changes in the brain of young rats. Brain Res Bull 2008;76(6):626-39.

9. Portugal G, Gould T. Genetic variability in nicotinic acetylcholine receptors and nicotine addiction: Coverging evidence from human and animal research. Behav Brain Res 2008;193(1):1-16.

10. Arredondo J, Chernyavsky A, Marubio L, Beaudet A, Jolkovsky D, Pinkerton K, Grando S. Receptor - Mediated Tobacco Toxicity. Regulation of gene expression through $\alpha 3 \beta 2$ nicotinic receptor in oral epithelial cells. Am J Pathol 2005;166(2): 597-613.

11. Marković D, Vucković N, Jefić B, Strajnić L, Mirković S. Effects of smoking on edentulous alveolar ridge mucosa. Med Preql 2005;58(1-2): 23-6.

12. Ali A. Histolpathologic changes in oral mucosa of Yemenis addicted to water-pipe and cigarette smoking in addition to takhzen al- qat. Oral Surg Oral Med Oral Pathol Oral Radiol Endod 2007; 103(3):e55-e59.

13. López-Blanc S, Collet A, Gandolfo M, Femopase F, Hernández S, Tomasi V, Paparella M, Itoiz M. Nucleolar organizar regions (AgNOR) and subepitelial vascularization as field cancerization markers in oral mucosa biopsias of alcoholic and smoking patients. Oral Surg Oral Med Oral Pathol Oral Radiol Endod 2009;108(5):747-53.

14. Sridhar S, Schembri F, Zeskind J, Shah V, Gustafson A, Steiling K, Liu G, Dumas Y, Zhang X, Brody J, Lengburg M, Spira A. Smoking-induced gene expression changes in the bronchial airway are reflected in nasal and buccal epithelium. BMC Genomics 2008;9:259:1-13.

15. Boyle J, Gümüş Z, Kacker A, Choksi V, Bocker J, Zhou X, Yantiss R, Hughes D, Du B, Judson B, Subbaramaiah K, Dannenberg A. Effects of Cigarette Smoke on the Human Oral Mucosal Transcriptome. Cancer Prev Res (Phila) 2010;3 (3):266-78.

16. Bánóczy J, Gintner Z, Dombi C. Tobacco use and oral leucoplakia. J Dent Educ 2001;65(4): 322-7.

17. Pentenero M, Giaretti W, Navone R, Rostan I, Gassino L, Broccoletti R, Arduino P, Malacarne D, Gandolfo S. Evidence for a possible anatomical subsite-mediated effect of tobacco in oral potentially malignant disorders and carcinoma. J Oral Pathol Med 2011;40(3):214-7.

18. Madani A, Dikshit M, Bhaduri D. Risk for oral cancer associated to smoking, smokeless and oral dip products. Indian J Public Health 2012;56 (1):57-60.

19. Wang Y, Wan Z, Zhou Y, Liu L, Zhao Y, Yao C, Wang L, Qiao Z. Nicotine stimulates adhesión molecular expresión via calcium influx and mitogen-activated proteína kinases in human endotelial cells. Int JBiochem Cell Biol 2006;38 (2):170-82.

20. Steptoe A, Ussher M. Smoking, cortisol and nicotine. Int J of Psychophysiol 2006;59(3):228-35.

21. Torres de Heens G, van der Velden U, Loos B. Cigarette smoking enhances $T$ cell activation and a Th2 inmune response; an aspecto of the pathophysiology in periodontal disease. Citokine 2009; 47(3):157-61.

22. Moszczyński P, Żabiński Z, Moszczyński P Jr, Rutowski J, Słowiński, Tabarowski Z. Inmunological findings in cigarette smokers. Toxicol Lett 2001; 118(3):121-7.

23. Wu P, Loh C, Hsieh L, Liu T, Chen C, Liou S. Clastogenic effect for cigarette smoking but not areca quid chewing an measured by micronuclei 
in exfoliated buccal mucosal cells. Mutat Res 2004; 562(1-2):27-38.

24. Scott D, Palmer R. The influence of tobacco smoking on adhesion molecule profiles. Tob Induc Dis 2002; 1(1):7-25.

25. Lee J, Cooke J. Nicotine and pathological angiogenesis. Life Sci 2012;91(21-22):1058-64.

26. Allam E, Delacruz K, Ghoneima A, Sun J, Windsor L. Effects of tobacco on cytokine expression from human endothelial cells. Oral Dis. 2012: 2012
Dec 1. doi: 10.1111/odi.12050. [Epub ahead of print].

\section{CORRESPONDENCIA}

Mariana Villarroel-Dorrego

Facultad de Odontología.

Universidad Central de Venezuela

Los Chaguaramos 1063

Caracas. Venezuela

Correo electrónico: mariana.villarroel@ucv.ve 